\title{
Spin-torque oscillation in large size nano-magnet with perpendicular magnetic fields
}

Linqiang Luo ${ }^{1}$, Mehdi Kabir ${ }^{2}$, Nam Dao ${ }^{3}$, Salinporn Kittiwatanakul ${ }^{3}$, Michael Cyberey ${ }^{4}$, Stuart A. Wolf ${ }^{1,3,5}$, Mircea $\operatorname{Stan}^{2}$ and Jiwei $\mathrm{Lu}^{3}$

${ }^{1}$ Department of Physics, University of Virginia, Charlottesville, Virginia, 22904, USA

${ }^{2}$ Department of Electrical \& Computer Engineering, University of Virginia, Charlottesville, Virginia, 22904, USA

${ }^{3}$ Department of Materials Science \& Engineering, University of Virginia, Charlottesville, Virginia, 22904, USA

${ }^{4}$ Department of Electrical Engineering, University of Virginia, Charlottesville, Virginia, 22904, USA

${ }^{5}$ Institute of Defense Analyses, Alexandria, Virginia, 22311, USA

Corresponding Author: Linqiang Luo, Email: LL6UK@ virginia.edu 


\begin{abstract}
DC current induced magnetization reversal and magnetization oscillation was observed in $500 \mathrm{~nm}$ large size $\mathrm{Co}_{90} \mathrm{Fe}_{10} / \mathrm{Cu} / \mathrm{Ni}_{80} \mathrm{Fe}_{20}$ pillars. A perpendicular external field enhanced the coercive field separation between the reference layer $\left(\mathrm{Co}_{90} \mathrm{Fe}_{10}\right)$ and free layer $\left(\mathrm{Ni}_{80} \mathrm{Fe}_{20}\right)$ in the pseudo spin valve, allowing a large window of external magnetic field for exploring the free-layer reversal. A magnetic hybrid structure was achieved for the study of spin torque oscillation by applying a perpendicular field $>3 \mathrm{kOe}$. The magnetization precession was manifested in terms of the multiple peaks on the differential resistance curves. Depending on the bias current and applied field, the regions of magnetic switching and magnetization precession on a dynamical stability diagram has been discussed in details. Micromagnetic simulations are shown to be in good agreement with experimental results and provide insight for synchronization of inhomogeneities in large sized device. The ability to manipulate spin-dynamics on large size devices could be proved useful for increasing the output power of the spin-transfer nano-oscillators (STNOs).
\end{abstract}

\title{
Keywords
}

Spin transfer torque switching; Spin torque oscillation; Nanostructure; Micromagnetic simulation; STNO power 


\section{Introduction}

Spin-polarized currents can be harnessed to manipulate magnetization and excite oscillation via the spin transfer torque (STT) effect, and are utilized in the application of MRAM[1, 2] and spin-transfer nano-oscillators (STNOs) [3, 4]. STNOs have the advantages that their frequencies are highly tunable by current and magnetic field over a range from a few $\mathrm{GHz}$ to $40 \mathrm{GHz}[3,5]$. Furthermore, the nanometer sized devices are among the smallest microwave oscillators yet developed[6] and their compatibility with standard silicon processing opens the possibility for on-chip applications [7, 8]. However, the bottlenecks for the widespread application of STNOs lies in the enhancement of the output power above the current limit of $\sim 0.5 \mu \mathrm{W}$ [9]. It has been suggested that two nano-contact STNOs in close proximity could mutually phase-lock and increase the output power; however phase-locking of more than two STNOs remains technologically

challenge [10-14]. Instead of putting an array of STNOs nano-magnets together, where the oscillation of the magnetic domain underneath those point-contacts were synchronized by the propagation of spin-waves and increased the output power [15], we 
propose to make use of larger sized magnets in the hope that synchronization of multiple domains could lead to higher output power, and firstly we demonstrated that spin-transfer torque could be used to efficiently induce magnetization switching and oscillation in 500 nm large size devices. For large size device, our simulation results have shown that the non-uniform oscillations tend to synchronize with each other and generate coherent oscillation.

\section{Materials and Methods}

The magnetic multilayer was synthesized by sputtering in a Biased Target Ion Beam

Deposition system (BTIBD). The deposition details can be found elsewhere [16]. The complete structure of the multilayer is $\mathrm{SiO}_{2}$ (substrate)/20nm $\mathrm{Ru} / 2.2 \mathrm{~nm} \mathrm{Co}_{90} \mathrm{Fe}_{10}$ (reference layer) $/ 5 \mathrm{~nm} \mathrm{Cu} / 6.5 \mathrm{~nm} \mathrm{Ni} \mathrm{Ni}_{80} \mathrm{Fe}_{20}$ (free layer) $/ 5 \mathrm{~nm} \mathrm{Ru} / \mathrm{Ti} 5 \mathrm{~nm} / \mathrm{Au} 25 \mathrm{~nm}$. A magnetoresistance (MR) of $\sim 1.2 \%$ was measured in the pseudo spin-valve continuous film using current-in-plane method before the patterning process [Fig. 1(a)]. The in-plane hysteresis loop revealed a good separation between the reference layer and free layer coercive fields of $30 \mathrm{Oe}$ and $5 \mathrm{Oe}$, respectively. Then the magneto-transport behavior was 
characterized with a field applied in the out-of-plane (OOP) direction [Fig. 1(b)]. By

applying an out-of-plane field, a much larger coercive field separation between the reference layer ( 800 Oe) and free layer $(\sim 100$ Oe) was achieved. The increase of the coercive fields can be understood in terms of the Stoner-Wohlfarth model [17].

The film was then patterned using photolithography and then ion milled to form a round pillar with a nominal cross-section diameter of $500 \mathrm{~nm}$. By monitoring the change of thickness and magnetization, we were able to precisely control the ion milling so that the $\mathrm{Co}_{90} \mathrm{Fe}_{10}$ layer was partially etched [as shown schematically in Fig. 1(c)]. With such geometry, the $\mathrm{Co}_{90} \mathrm{Fe}_{10}$ reference layer is relatively insensitive to the spin transfer torques due to its extended volume. Fig. 1(d) shows a scanning electron microscope image of the $500 \mathrm{~nm}$-diameter size pillar. To reduce the resistance of the transmission lines, a very thick Au layer $(4 \mu \mathrm{m})$ was electro-deposited on the top contact areas, serving as the top bonding pads, as seen in the optical microscope image Fig. 1(e). The differential resistance is measured using a lock-in amplifier circuit in the PPMS system (PPMS 6000, Quantum Design). Positive current is defined in a direction such that electrons flow from 
$\mathrm{Co}_{90} \mathrm{Fe}_{10}$ to $\mathrm{Ni}_{80} \mathrm{Fe}_{20}$. All transport measurements reported in this work were obtained at room temperature. Micromagnetic simulations were carried out based on the LLG equation including a spin-torque term at zero temperature [18]. The boundary condition of the modeling was defined based on the dimension of a nano-pillar, which is a perfect circular shape with a diameter of $500 \mathrm{~nm}$. The parameters used to model the free layer were $M_{S}=650 \mathrm{emu} / \mathrm{cm}^{3}$, exchange stiffness constant of $A=1.3 \times 10^{-6} \mathrm{erg} / \mathrm{cm}$, and damping constant of $\alpha=0.009$. The mesh size was $10 \times 10 \times 3.25 \mathrm{~nm}^{3}$. The spin polarization parameter $P$ was assumed to be 0.4 .

\section{Results and discussion}

The differential resistance $d V / d I$ versus perpendicular applied magnetic field $H$ is plotted in Fig. 1(f). The layers switch between antiparallel and parallel alignment with a current perpendicular to the plane (CPP) GMR ratio of $1.0 \%$. The slightly lower GMR ratio after patterning might be due to the lead resistance that was in series with the pillar while it did not contribute to the MR. The coercive field of both layers increased significantly over the continuous film values. The coercive field of the $\mathrm{Co}_{90} \mathrm{Fe}_{10}$ reference 
layer was $\sim 6.6 \mathrm{kOe}$ while the $\mathrm{Ni}_{80} \mathrm{Fe}_{20}$ free layer had a coercive field of $\sim 1.3 \mathrm{kOe}$. This is in accordance with observations on patterned nano-magnets, where the coercive field increases as it transitions from reversal through domain nucleation and domain wall motion in a full film towards coherent rotation in patterned structures [19]. As aforementioned, the reference layer had a significantly higher coercive field, allowing a large window of external magnetic fields for only the free-layer reversal. The gradual increase in resistance that occurs in Fig. 1(c) from 12kOe to 0Oe is from the anisotropic magnetoresistance (AMR) due to the remaining $\mathrm{Co}_{90} \mathrm{Fe}_{10}$ continuous film.

While both layers' magnetization tends to rotate toward the out-of-plane direction under the perpendicular field, the $\mathrm{Ni}_{80} \mathrm{Fe}_{20}$ layer's magnetization can be pulled more easily out of plane due to its smaller demagnetizing field $\left(4 \pi M_{S}\right)$. In comparison, a much larger external field was required to rotate the $\mathrm{Co}_{90} \mathrm{Fe}_{10}$ layer's magnetization. To confirm, the rotation angle curves were measured for both single layer $\mathrm{Ni}_{80} \mathrm{Fe}_{20}$ [Fig.2 blue] and single layer $\mathrm{Co}_{90} \mathrm{Fe}_{10}$ [Fig.2 red]. The rotation angle curve basically tells the information how big the angle of the magnetization rotates towards the out-of-plane direction. Both 
the $\mathrm{Ni}_{80} \mathrm{Fe}_{20}$ and $\mathrm{Co}_{90} \mathrm{Fe}_{10}$ layer' magnetization has been pulled out-of-plane at $30 \mathrm{kOe}$

field. As the field decreases, the $\mathrm{C}_{90} \mathrm{Fe}_{10}$ layer's magnetization first starts to relax toward in-plane direction due to its large demagnetizing field. At $\sim 9 \mathrm{kOe}$, there is a maximum angle difference $\sim 50^{\circ}$ between the two layers' magnetization. The relative angle of the two layer's magnetization will then decrease with the further decreasing of the field and eventually reach 0 or $180^{\circ}$ as both layers relax back in-plane. As a result, a pseudo hybrid configuration with an out-of-plane free layer and a near in-plane reference layer can be achieved at high perpendicular fields from $\sim 3 \mathrm{kOe}$ to $\sim 9 \mathrm{kOe}$. The magnetic hybrid configuration has been predicted to be more efficient at generating large amplitude precession and therefore increases the output power in STNOs [20-22].

Prior to the magneto-transport measurements, the sample was placed in an out-of-plane field of $30 \mathrm{kOe}$, and then the field was set to the specific values. In Fig. 3(a), we show the variation of the differential resistance $d V / d I$ versus injected DC current at small fields. At $H=0$, starting from the parallel (P) state at zero current, the DC current first decreased toward the negative direction. A jump in the differential resistance was 
observed at $I_{C}^{P-A P}=-48 \mathrm{~mA}$ (current density of $2.45 \times 10^{7} \mathrm{~A} / \mathrm{cm}^{2}$ ) due to the magnetization switching which occurred in the $\mathrm{Ni}_{80} \mathrm{Fe}_{20}$ layer. The curve showed hysteretic behavior because the system remained in the antiparallel (AP) state until the current was swept back to a positive critical current of $I_{C}^{A P-P}=47 \mathrm{~mA}$ (current density of $2.39 \times 10^{7} \mathrm{~A} / \mathrm{cm}^{2}$ ), where the resistance dropped back to the $\mathrm{P}$ state. The corresponding change in the differential resistance was $\sim 95 \mathrm{~m} \Omega$ for both negative and positive switching, which was of the same magnitude of change in resistance shown in the MR minor loop $\sim 0.1 \Omega$ [Fig. 3(a) inset]. Therefore, it was certain that the experimental hysteretic curve was caused by spin torque induced magnetization reversal typically observed at zero or low applied magnetic field [3]. The hysteretic reversal here was fundamentally different from the switching by the Oersted field that was self-generated by the current passing through the pillar [23]. The magnitude of the Oersted field was independent of the current direction, which would have resulted in a symmetric $d V / d I$ curve with respect to current. Note that the overall parabolic increase in $d V / d I$ can be ascribed to the electron scattering by emissions of phonons and magnons in metallic point contacts [24]. 
Micromagnetic simulations qualitatively reproduced the dynamic process of the current induced switching observed experimentally. Fig. 3(b) shows a typical switching from AP to P reproducing the behavior shown in the Fig. 3(a) blue curve. For our device, the Oersted field is not strong enough to form a full vortex at the experimental currents used ( 48 mA). The micromagnetic simulation suggests a formation of a full vortex by the Oersted field requires a current of at least $\sim 100 \mathrm{~mA}$, which agrees with a previous report [25]. Instead, a $C$-shape magnetization state tends to form under the Oersted fields, as shown in Fig. 3(b). The $C$-state has a majority magnetization pointing along the parallel or anti-parallel direction. Under the action of the spin-transfer torque, the $C$-state rotates toward the opposite direction leading to the switching from antiparallel to parallel.

Starting from a parallel alignment of the magnetic layers, a series of $d V / d I$ versus current density $J_{B}$ scans were measured at different fields to construct the $J_{B}-H$ dynamic stability diagram shown in Fig. 4(a). Each symbol in the diagram corresponds to a discrete change in the resistance while changing $J_{B}$ at a corresponding field. We discuss the results in Fig. 4 in details below; the general features of the dynamic stability diagram 
are also seen in $130 \mathrm{~nm} \times 70 \mathrm{~nm} \mathrm{Co} / \mathrm{Cu} / \mathrm{Co}$ devices [3]. Under $3.2 \mathrm{kOe}$, we observe hysteretic reversal of magnetization between the AP and $\mathrm{P}$ states where the red circles correspond to the critical current for AP-P transition $J_{C}{ }^{A P-P}$ and blue squares correspond to the critical current density for P-AP transition $J_{C}{ }^{P-A P}$. For $H>3.2 \mathrm{kOe}$ we observe magnetic transitions that are reversible in $I_{B}$ scans and give sharp peaks in $d V / d I$. The peaks were demonstrated in Fig. 4(b) with the $d V / d I$ curve recorded at $H=3.5$ kOe. The differential resistance measurements $(d V / d I)$ can be generally expressed:

$$
\frac{d V}{d I}=\frac{d\left(I R d_{\text {.c. }}\right)}{d I}=R d . c .+I \frac{d R d . c .}{d I}
$$

Assuming that the system is ohmic such that $d R d . c . d I I=0$, then $d V / d I=R$ d.c. . However, for the spin torque oscillator, it was equivalent to an inductor-capacitor-resistor (LCR) circuit [26]. Therefore, the second term, $I R$ d.c. $/ d I$, needed to be included and can add or subtract to $R$ d.c. depending on the sign of $I$ and the sign of the slope $(d R d . c / d I)$. In Fig. 4(b), the slope of $R$ d.c. versus $I$ (e.g. $d R R_{d . c} / d I$ ) was negative. And the sign of $I$ was also negative giving peaks on $d V / d I$.

For $H<3.2 \mathrm{kOe}$, the change of critical current density $J_{C}{ }^{A P-P}$ follows a linear $H$ 
dependence. This agrees with the critical current density equations given by the Slonczewski model [27, 28]:

$$
J_{C} \approx \frac{A \alpha M_{S} d}{g(\theta) \mathrm{P}}\left(-H_{K}+4 \pi M_{S}-H_{d i p}-H\right)
$$

where $M_{s}, d$ and $\alpha$ are the saturation magnetization, thickness and Gilbert damping constant for the free layer, respectively. $A$ is a constant coefficient of the order of $10^{11} \mathrm{~mA}$ $\mathrm{Oe}^{-1} \mathrm{emu}^{-1}, P$ is the spin polarization, $g(\theta)$ is a scalar factor depending on the relative angle of the reference layer and free layer magnetizations [28]. $H, H_{d i p}$ and $H_{k}$ are the perpendicular field, the dipolar field and the anisotropy field, respectively, while $4 \pi M_{s}$ arises from the demagnetizing field. $J_{C}^{A P-P}$ follows the linear $H$ dependence with a slope $=-1.66 \times 10^{4} \mathrm{~A} /\left(\mathrm{Oe} \cdot \mathrm{cm}^{-2}\right)$ and intercepts $J_{B}=0$ near $2 \mathrm{kOe}$, which is roughly the positive coercive field as shown in Fig. 3(a) inset. By extracting the slope of $J_{C}{ }^{A P-P}$ and comparing with the pre-factor in Eq. (2), we estimate $\alpha / P=0.42 g(\pi)$. Given that $\alpha=0.009$ and $P=$ $0.4,[29]$ then $g(\pi)=0.054$, which is almost an order smaller than the expected range between 0.1 and 0.5 [30]. The difference might be associated with the multiple domains in our large size device. According to the Slonczewski model, Eq. (2) is strictly valid for 
single domain structure at low temperatures [28]. Hence, Eq. (2) may not be directly applicable to the possible multi-domain structures in a $500 \mathrm{~nm}$ magnetic nanopillar.

For $H>3.2 \mathrm{kOe}$, the hysteretic switching of the differential resistance is replaced by the peaks given by the green triangle symbols in the dynamic stability diagram. It is found that these peaks are generated only on the negative currents direction which corresponding to the electrons flow direction from $\mathrm{Ni}_{80} \mathrm{Fe}_{20}$ to $\mathrm{Co}_{90} \mathrm{Fe}_{10}$. This feature is also a signature of the spin transfer oscillation [31]. The change from irreversible hysteretic switching to reversible sweeping of the differential curve has been associated in standard nanopillars to the sustained precession of magnetization $[3,5]$.

In the dynamic stability diagram, the $J_{C}^{P-A P}$ and $J_{C}^{P-A P}$ curves are extrapolated to construct the oscillation regime [the blue regime shown in Fig. 4(a)]. Note that the oscillation regime also agrees with the magnetic field window where the magnetic hybrid configuration is achieved. The peaks generally shift to higher current with increasing fields following the trend of the extrapolated curve. Examining Fig. 4(b), we observe multiple peaks at $3.5 \mathrm{kOe}$. Furthermore, these peaks have different critical currents and 
show distinct amplitudes as shown in the second derivative curve Fig. 4(b) inset. Given the large size of our device, inhomogeneities, such as multiple domains, exist on the ferromagnetic layers. However, the micromagnetic simulations showed that the multiple domains tended to synchronize with each other under certain currents and led to a harmonic precession as demonstrated in Fig. 5(a). A non-coherent multiple domain precession occurred at the beginning Fig. 5(b) and evolved into a coherent oscillation as shown in Fig. 5(c). The coherent oscillation persisted for the rest of the time until the current bias was removed. For the simulation, the initial condition does not have a large effect on the synchronization process since the equilibrium state was extracted firstly and was used as the starting condition for the current induced dynamics simulation. For example, for Fig. 5a, a magnetic equilibrium state under the external field of 3.5kOe was first extracted from the micromagnetic simulation with zero current density. Then this equilibrium state was used as the starting condition for the simulation under different non-zero current densities. This also mimics the experiments, where an out of plane magnetic field was applied to the nano-pillar (to achieve the pseudo hybrid configuration) 
before applying the bias current. Based on the simulation, the multiple peaks are likely to be associated with different spin-wave modes that are represented by the different precession orbits as shown in Fig. 5(d). Depending on the external fields, at those different current densities, there were different metastable states where the spin transfer torque term could balance out the damping term leading to stable precession orbits. The orbits shown are numerically calculated from the LLGS equation with out-of-plane field of 3.5kOe. According to Eq. (1), the amplitude of the peaks shown in Fig. 4(b) were mainly determined by the second term $I d R d . c / d I$ and reflected the change of $M R$.. This also qualitatively agreed with the simulation results shown in Fig. 5(d), where a higher current density $\left(3.40 \times 10^{7} \mathrm{~A} / \mathrm{cm}^{2}\right)$ resulted a larger orbit, hence a larger change of the $M R$ during the precession. A large external field could suppress the number of domains and possibly lead to the single mode precession as shown in the dynamic stability diagram for $H=4.5 \mathrm{kOe}$ and $5 \mathrm{kOe}$, yet, more work needed to be carried out to confirm the synchronizations experimentally on the frequency domain [32,33].

\section{Conclusions}


In summary, $500 \mathrm{~nm}$ large size $\mathrm{Co}_{90} \mathrm{Fe}_{10} / \mathrm{Cu} / \mathrm{Ni}_{80} \mathrm{Fe}_{20}$ pseudo spin valve pillars were

fabricated using only photolithographic techniques. We demonstrate the magnetization reversal and magnetization oscillation on these large sized devices. At large fields, only the negative polarity currents cause peaks in the $d V / d I$, which is consistent with the excitation of precessing spin wave modes. The critical current for the magnetization switching and oscillation have been plotted together to construct the dynamic stability diagram. In contrast to the dynamic diagrams of standard nanopillars, the switching regime of our devices show deviation from the Slonsczewski model, which might be due to the multiple domains of our large size pillar. Our simulations indicate that the multiple peaks could come from the different spin-wave modes with independent precession orbits.

\section{Acknowledgement}

Authors are grateful to the support from the National Science Foundation (Award No. ECCS-1344218). J.L. thanks the support from C-SPIN center.

\section{References:}

[1] C. Chappert, A. Fert, F.N. Van Dau, The emergence of spin electronics in data storage, 
Nat Mater, 6 (2007) 813-823.

[2] R. Sbiaa, S.Y.H. Lua, R. Law, H. Meng, R. Lye, H.K. Tan, Reduction of switching current by spin transfer torque effect in perpendicular anisotropy magnetoresistive devices (invited), Journal of Applied Physics, 109 (2011) 07C707.

[3] S.I. Kiselev, J.C. Sankey, I.N. Krivorotov, N.C. Emley, R.J. Schoelkopf, R.A. Buhrman, D.C. Ralph, Microwave oscillations of a nanomagnet driven by a spin-polarized current, Nature, 425 (2003) 380-383.

[4] L. Berger, Emission of spin waves by a magnetic multilayer traversed by a current, Physical Review B, 54 (1996) 9353-9358.

[5] W.H. Rippard, M.R. Pufall, S. Kaka, S.E. Russek, T.J. Silva, Direct-Current Induced Dynamics in CoFe/NiFe Point Contacts, Physical Review Letters, 92 (2004) 027201.

[6] P. Villard, U. Ebels, D. Houssameddine, J. Katine, D. Mauri, B. Delaet, P. Vincent, M.C. Cyrille, B. Viala, J.P. Michel, J. Prouvee, F. Badets, A GHz Spintronic-Based RF Oscillator, IEEE Journal of Solid-State Circuits, 45 (2010) 214-223.

[7] S.E. Russek, W.H. Rippard, T. Cecil, R. Heindl, Handbook of Nanophysics: Functional Nanomaterial ch.38 p.1, CRC Press, 2010.

[8] M. Kabir, M. Stan, Synchronized Spin Torque Nano-Oscillators: From Theory to Applications, in: W. Zhao, G. Prenat (Eds.) Spintronics-based Computing, Springer International Publishing, Cham, 2015, pp. 231-250.

[9] Z. Zeng, P.K. Amiri, I.N. Krivorotov, H. Zhao, G. Finocchio, J.-P. Wang, J.A. Katine, Y. Huai, J. Langer, K. Galatsis, K.L. Wang, H. Jiang, High-Power Coherent Microwave Emission from Magnetic Tunnel Junction Nano-oscillators with Perpendicular Anisotropy, ACS Nano, 6 (2012) 6115-6121.

[10] A.M. Deac, A. Fukushima, H. Kubota, H. Maehara, Y. Suzuki, S. Yuasa, Y. Nagamine, K. Tsunekawa, D.D. Djayaprawira, N. Watanabe, Bias-driven high-power microwave emission from $\mathrm{MgO}$-based tunnel magnetoresistance devices, Nature Physics, 4 (2008) 803-809.

[11] A. Ruotolo, V. Cros, B. Georges, A. Dussaux, J. Grollier, C. Deranlot, R. Guillemet, K. Bouzehouane, S. Fusil, A. Fert, Phase-locking of magnetic vortices mediated by antivortices, Nature Nanotechnology, 4 (2009) 528-532. 
[12] S. Kaka, M.R. Pufall, W.H. Rippard, T.J. Silva, S.E. Russek, J.A. Katine, Mutual phase-locking of microwave spin torque nano-oscillators, Nature, 437 (2005) 389-392.

[13] F.B. Mancoff, N.D. Rizzo, B.N. Engel, S. Tehrani, Phase-locking in double-point-contact spin-transfer devices, Nature, 437 (2005) 393-395.

[14] S. Sani, J. Persson, S.M. Mohseni, Y. Pogoryelov, P. Muduli, A. Eklund, G. Malm, M. Käll, A. Dmitriev, J. Åkerman, Mutually synchronized bottom-up multi-nanocontact spin-torque oscillators, Nature communications, 4 (2013).

[15] M. Madami, S. Bonetti, G. Consolo, S. Tacchi, G. Carlotti, G. Gubbiotti, F. Mancoff, M.A. Yar, J. Åkerman, Direct observation of a propagating spin wave induced by spin-transfer torque, Nature nanotechnology, 6 (2011) 635-638.

[16] L. Luo, N. Anuniwat, N. Dao, Y. Cui, S.A. Wolf, J. Lu, Magneto-transport and domain wall scattering in epitaxy L10 MnAl thin film, Journal of Applied Physics, 119 (2016) 103902.

[17] C. Tannous, J. Gieraltowski, The Stoner-Wohlfarth model of ferromagnetism, European Journal of Physics, 29 (2008) 475.

[18] M. Donahue, D. Porter, Oommf user's guide, version 1.0, interagency report nistir 6376, National Institute of Standards and Technology, (1999).

[19] G. Hu, T. Thomson, C.T. Rettner, S. Raoux, B.D. Terris, Magnetization reversal in CoPd nanostructures and films, Journal of Applied Physics, 97 (2005) $10 \mathrm{~J} 702$.

[20] W.H. Rippard, A.M. Deac, M.R. Pufall, J.M. Shaw, M.W. Keller, S.E. Russek, G.E. Bauer, C. Serpico, Spin-transfer dynamics in spin valves with out-of-plane magnetized CoNi free layers, Physical Review B, 81 (2010) 014426.

[21] Z. Zeng, G. Finocchio, B. Zhang, P. Khalili Amiri, J.A. Katine, I.N. Krivorotov, Y. Huai, J. Langer, B. Azzerboni, K.L. Wang, H. Jiang, Ultralow-current-density and bias-field-free spin-transfer nano-oscillator, Sci Rep, 3 (2013) 1426.

[22] H. Kubota, K. Yakushiji, A. Fukushima, S. Tamaru, M. Konoto, T. Nozaki, S. Ishibashi, T. Saruya, S. Yuasa, T. Taniguchi, Spin-torque oscillator based on magnetic tunnel junction with a perpendicularly magnetized free layer and in-plane magnetized polarizer, Applied Physics Express, 6 (2013) 103003.

[23] K. Bussmann, G.A. Prinz, S.-F. Cheng, D. Wang, Switching of vertical giant 
magnetoresistance devices by current through the device, Applied Physics Letters, 75 (1999) 2476-2478.

[24] A.G.M. Jansen, A.P.v. Gelder, P. Wyder, Point-contact spectroscopy in metals, Journal of Physics C: Solid State Physics, 13 (1980) 6073.

[25] J.P. Strachan, V. Chembrolu, Y. Acremann, X.W. Yu, A.A. Tulapurkar, T. Tyliszczak, J.A. Katine, M.J. Carey, M.R. Scheinfein, H.C. Siegmann, J. Stohr, Direct observation of spin-torque driven magnetization reversal through nonuniform modes, Phys Rev Lett, 100 (2008) 247201.

[26] M.R. Stan, M. Kabir, S. Wolf, J. Lu, Spin torque nano oscillators as key building blocks for the systems-on-chip of the future, in: Proceedings of the 2014 IEEE/ACM International Symposium on Nanoscale Architectures, ACM, 2014, pp. 37-38.

[27] J.C. Slonczewski, Current-driven excitation of magnetic multilayers, J Magn Magn Mater, 159 (1996) L1-L7.

[28] J.C. Slonczewski, Currents and torques in metallic magnetic multilayers, J Magn Magn Mater, 247 (2002) 324-338.

[29] A.S. Jenkins, R. Lebrun, E. Grimaldi, S. Tsunegi, P. Bortolotti, H. Kubota, K. Yakushiji, A. Fukushima, G. de Loubens, O. Klein, S. Yuasa, V. Cros, Spin-torque resonant expulsion of the vortex core for an efficient radiofrequency detection scheme, Nat Nanotechnol, 11 (2016) 360-364.

[30] S. Mangin, D. Ravelosona, J.A. Katine, M.J. Carey, B.D. Terris, E.E. Fullerton, Current-induced magnetization reversal in nanopillars with perpendicular anisotropy, Nat Mater, 5 (2006) 210-215.

[31] M. Tsoi, A.G.M. Jansen, J. Bass, W.C. Chiang, M. Seck, V. Tsoi, P. Wyder, Excitation of a Magnetic Multilayer by an Electric Current, Physical Review Letters, 80 (1998) 4281-4284.

[32] D.P. Bernstein, B. Bräuer, R. Kukreja, J. Stöhr, T. Hauet, J. Cucchiara, S. Mangin, J.A. Katine, T. Tyliszczak, K.W. Chou, Nonuniform switching of the perpendicular magnetization in a spin-torque-driven magnetic nanopillar, Physical Review B, 83 (2011) 180410.

[33] Y.-T. Cui, G. Finocchio, C. Wang, J. Katine, R. Buhrman, D. Ralph, Single-shot 
time-domain studies of spin-torque-driven switching in magnetic tunnel junctions, Physical review letters, 104 (2010) 097201. 


\section{Figure captions:}

Fig 1. (a) \& (b) Current in-plane (CIP) magnetoresistance (MR) curve on the full film before patterning with in-plane field or out-of-plane field respectively. (c) Schematic representation of the patterned pseudo-spin-valve sample. The bottom $\mathrm{Co}_{90} \mathrm{Fe}_{10}$ layer is partially patterned. The arrow shows the electron flow direction for negative current. (d) A SEM image of the patterned pillar with a diameter of $500 \mathrm{~nm}$. (e) The optical microscope image of a device with CPW contacts. (f) Current perpendicular to plane (CPP) MR curve on the patterned sample. The black arrows show the direction of the field sweep.

Fig 2. (Color online) Rotation angle curves for structure $20 \mathrm{~nm} \mathrm{Ru} / 6.5 \mathrm{~nm} \mathrm{Ni}{ }_{80} \mathrm{Fe}_{20} / 5 \mathrm{~nm} \mathrm{Ru}$ (blue triangles) and structure $20 \mathrm{~nm} \mathrm{Ru} / 2.2 \mathrm{~nm} \mathrm{Co} \mathrm{C}_{0} \mathrm{Fe}_{10} / 5 \mathrm{~nm} \mathrm{Ru}$ (red dots). The schematic diagrams show the relative magnetic configuration for the two layers. The black arrows indicate the position for the maximum angle difference between the two layers' magnetization direction.

Fig 3. (a) Differential resistance versus biased direct current $\left(I_{B}\right)$ with out-of-plane magnetic fields of 0 (bottom, red) and 200 Oe (blue). The current sweep starts at $0 \mathrm{~mA}$ and the arrows indicate the direction of the current sweep. The inset shows the minor MR hysteresis loop for $\mathrm{Ni}_{80} \mathrm{Fe}_{20}$ layer. The black arrows indicate the direction of the field sweep. (b) Micromagnetic simulation showing the evolution of $m_{x}$ component at a current density of $3 \times 10^{7} \mathrm{~A} / \mathrm{cm}^{2}$ with an out-of-plane field $H=200$ Oe. The flip-over of a $C$-state is demonstrated by the spatial magnetization distribution $1 \rightarrow 2 \rightarrow 3 \rightarrow 4$. The cores are added for illustration purpose.

Fig 4. (a) Experimentally determined $J_{B}-H$ (Out-of-Plane field) dynamic stability diagram for the $500 \mathrm{~nm}$ pillar device where each symbol corresponds to a distinct change in the resistance. Below $3.2 \mathrm{kOe}$, the symbols are from hysteretic switching where the circles (red) are $J_{C}^{A P-P}$ and squares (blue) are $J_{C}{ }^{P-A P}$. For field larger than $3.2 \mathrm{kOe}$, the triangles (green) refer to the peaks on the differential resistance curves. (b) $d V / d I$ versus $I_{B}$ for $H=3.5 \mathrm{kOe}$ corresponding to the field position marked by the dashed line in (a). The inset shows the 
second derivative of $d V / d I$ indicating the relative amplitude, width and position of the peaks.

Fig 5. (Color online) (a) Micromagnetic simulation showing the temporal magnetization component evolutions of $m_{x}$ (red), $m_{y}$ (blue) under an out-of-plane field of $3.5 \mathrm{kOe}$ and with a constant current density of $3.0 \times 10^{7} \mathrm{~A} / \mathrm{cm}^{2}$. (b)\&(c) The evolution of the spatial magnetization distribution at the corresponding time marked under them. (d) At $H=3.5$ $\mathrm{kOe}$, different current density leads to distinct stabilized precession orbits and frequencies. The main precession frequency $f$ is determined by taking the Fourier transforms of $m_{x}$. At $2.0 \times 10^{7} \mathrm{~A} / \mathrm{cm}^{2}$ (black), $3.0 \times 10^{7} \mathrm{~A} / \mathrm{cm}^{2}$ (red) and $3.4 \times 10^{7} \mathrm{~A} / \mathrm{cm}^{2}$ (blue), the simulated oscillation has the frequency of $3.15 \mathrm{GHz}, 10.5 \mathrm{GHz}$ and $14.6 \mathrm{GHz}$ respectively. 
Fig. 1
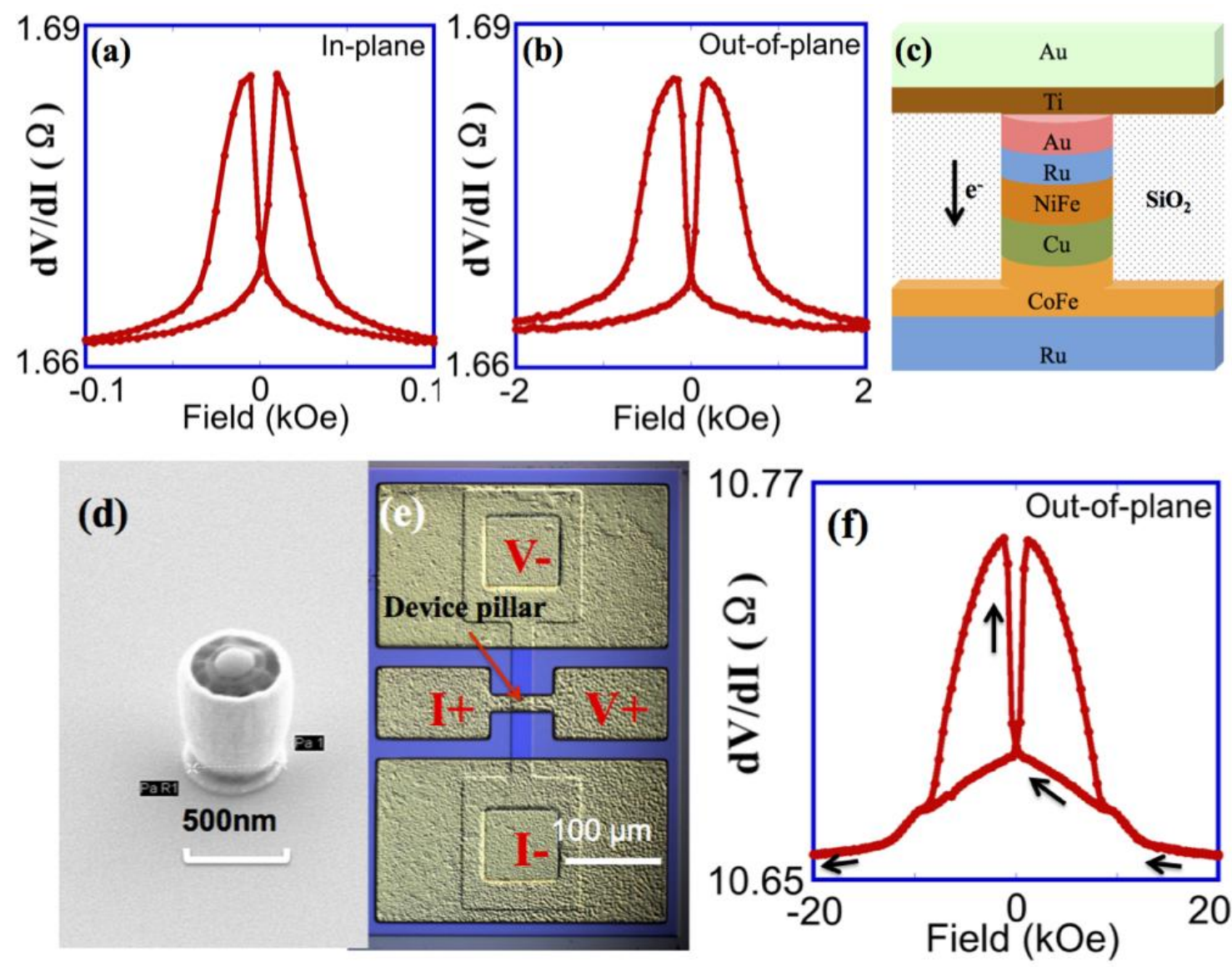
Fig. 2

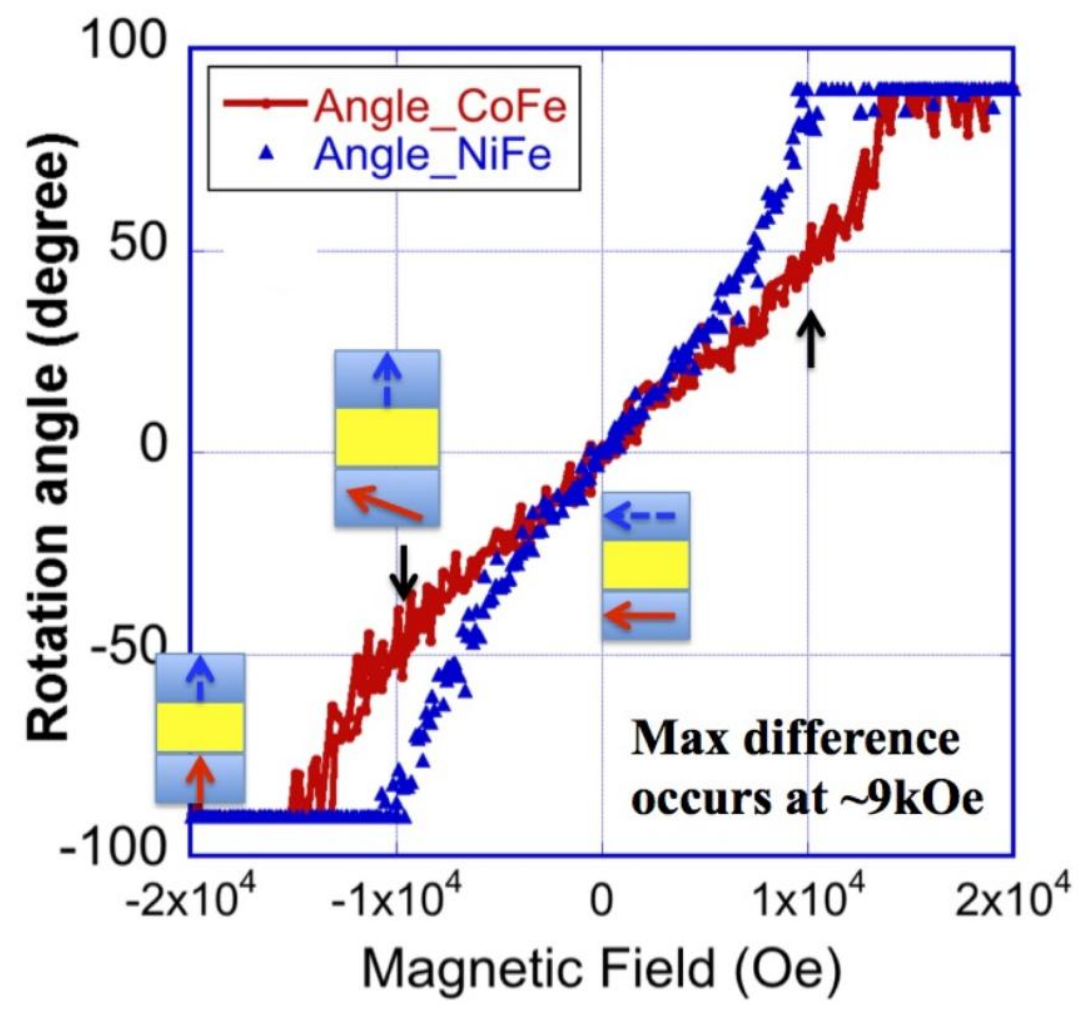


Fig. 3
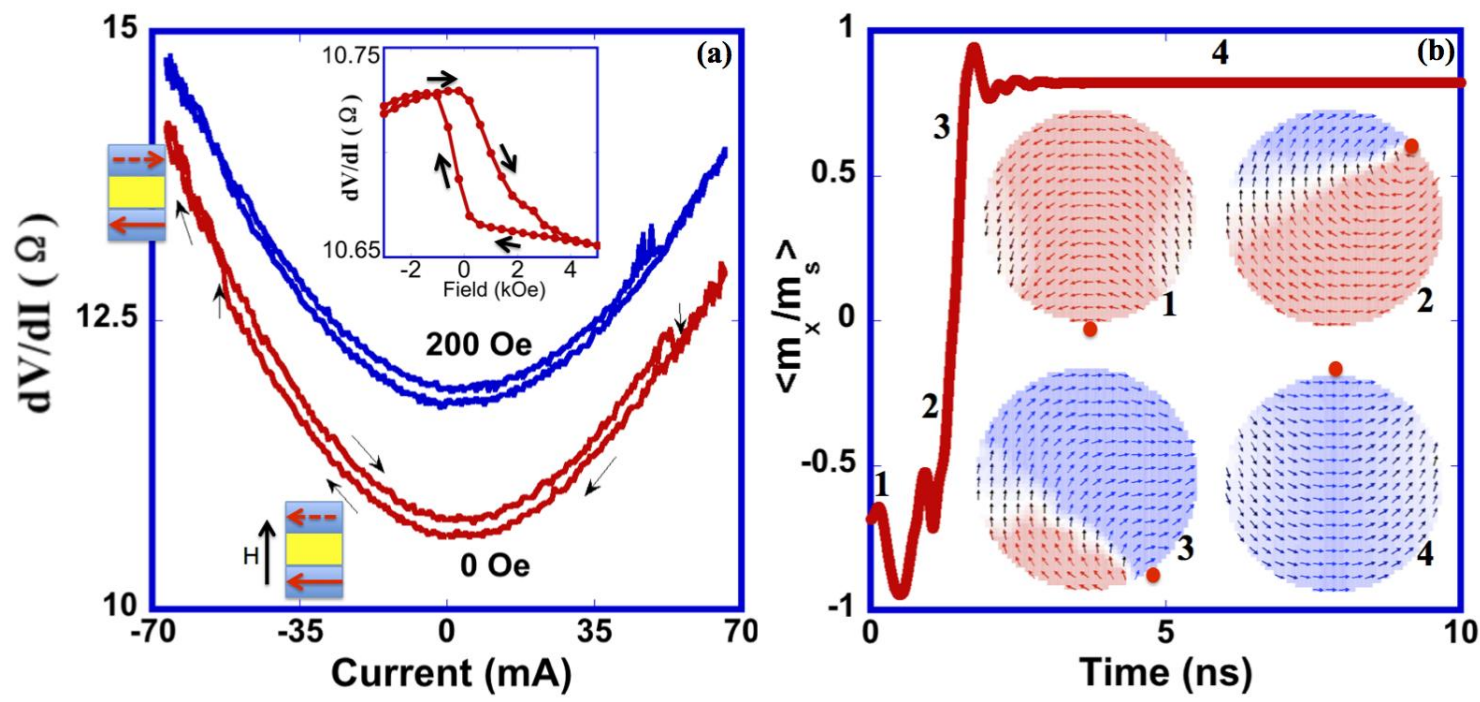

Fig. 4
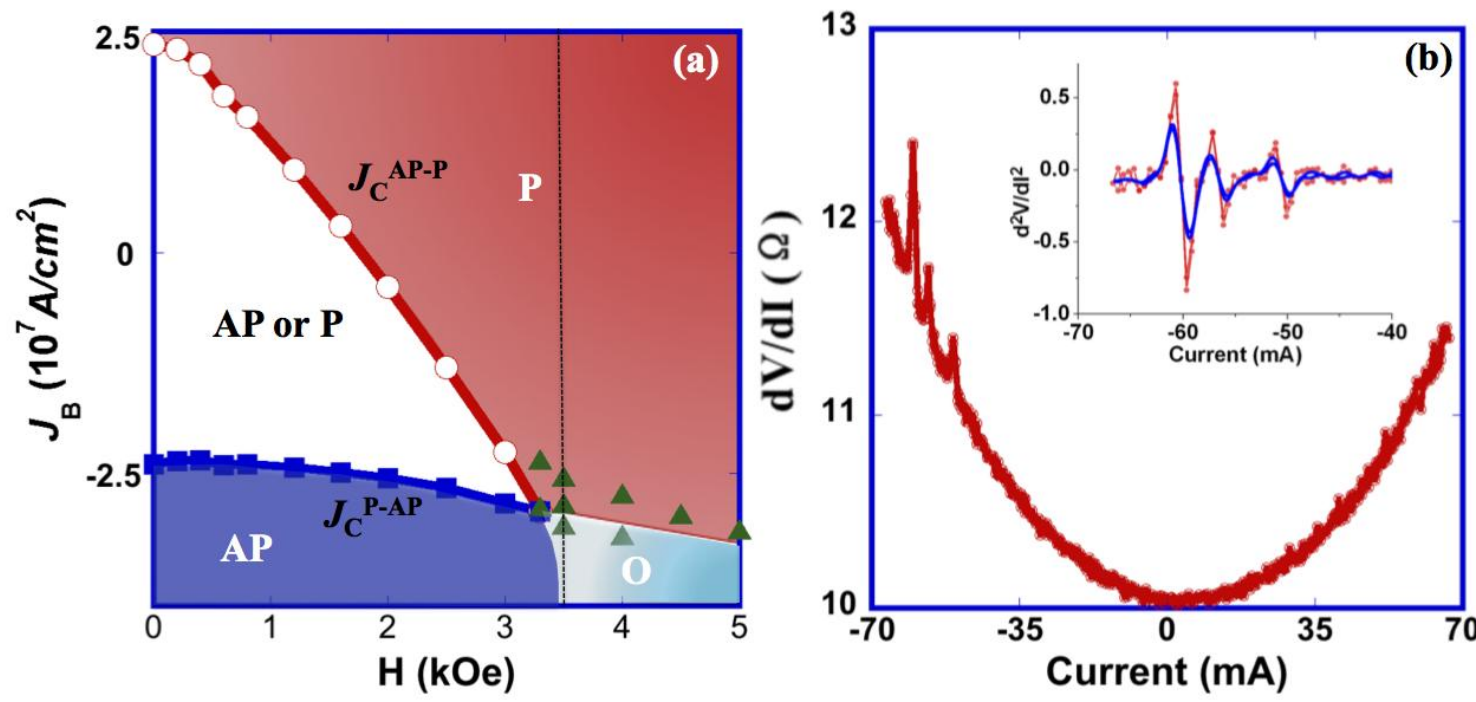
Fig. 5
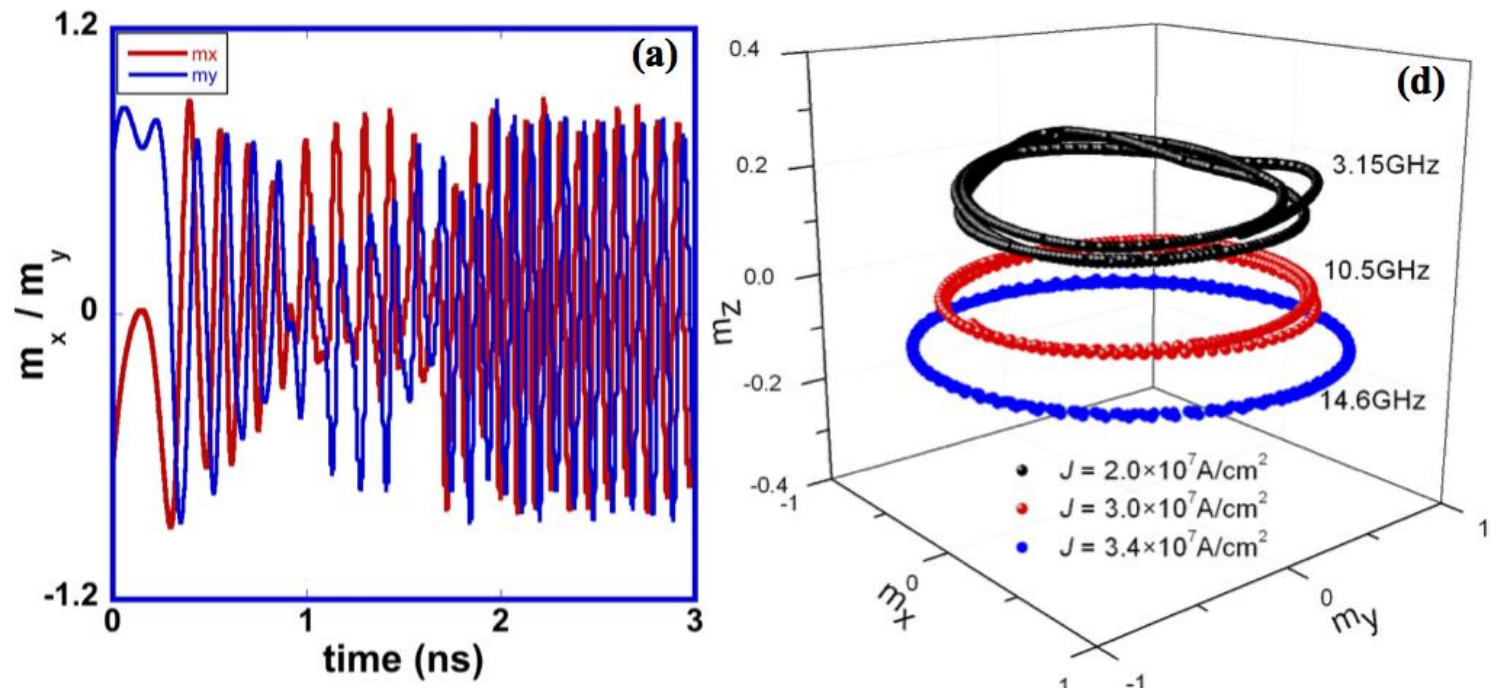

b)
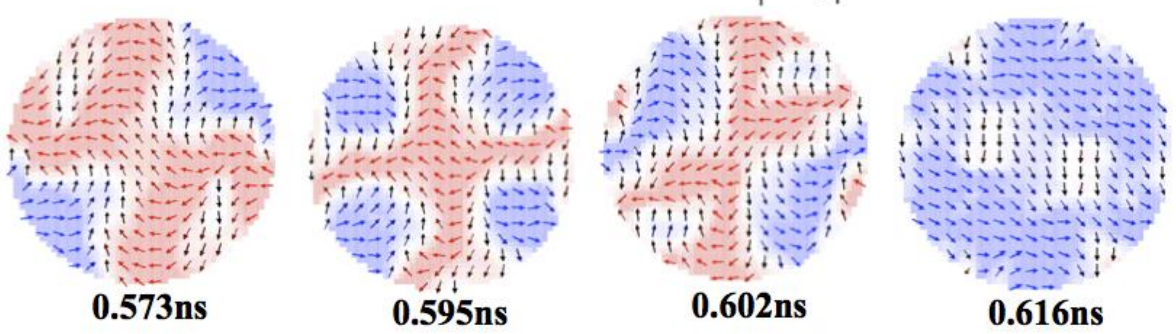

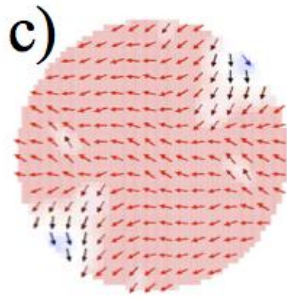

2.178ns

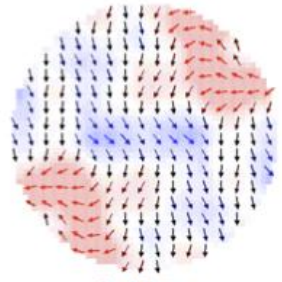

2.195ns

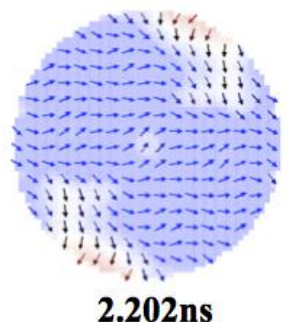

2.202ns
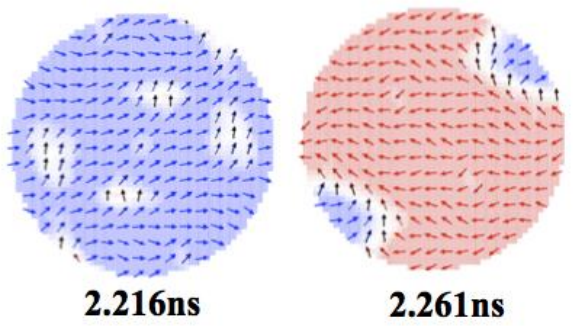